\title{
$\underline{P-51}$
}

\section{Chemical Constituents, Antibacterial and Antibiofilm Potential of the Ethanolic Extract of Melastoma Malabathricum}

\author{
Mohamad Shukri Kamaruddin ${ }^{1,}$, Nurthaharah Binti Idris ${ }^{1}$ and Mohd Fakharul Zaman Raja Yahya ${ }^{2}$ \\ ${ }^{1}$ School of Chemistry, Faculty of Applied Sciences SciencesUiTM Shah Alam, 40450 Shah Alam Selangor; ${ }^{2}$ School of \\ Biology, Faculty of Applied Sciences UiTM Shah Alam, 40450 Shah Alam Selangor; \\ E-mail: shukri_kamarudin@salam.uitm.edu.my
}

Melastoma malabathricum is a small shrub commonly found in cleared land, waste places, and roadside throughout the Southeast Asian countries, including Malaysia. Local name for this plant is "senduduk merah". The aim of the study was to investigate the phytochemical compounds, antibacterial and antibiofilm activity of the ethanolic extract of M. malabathricum. Phytochemical analysis of $M$. malabathricum leaves revealed the presence of tannins, alkaloids, saponins, phenols and terpenoids. No alkaloid was detected. In the GC-MS analysis, 10 bioactive phytochemical compounds were identified in the ethanolic extract of M. malabathricum. The main volatile compounds were squalene $(25 \%)$, hexadecanoic acid, ethyl ester $(18.95 \%)$, linolenic acid ethyl ester $(17.35 \%)$ and phytol $(11.89 \%)$. Microbroth dilution method showed that of $M$. malabathricum ethanolic extract against Pseudomonas aeruginosa strain ATCC 10145 was $6.25 \mathrm{mg} / \mathrm{ml}$. From disc diffusion assay, all test concentrations exhibited zone of inhibition against $P$. aeruginosa, which ranged from 9 to $11 \mathrm{~mm}$. Furthermore, it was also observed that $M$. malabathricum ethanolic extract inhibited formation of $P$. aeruginosa biofilm whereby numbers of all the test concentrations $\left(275-1650 \times 10^{3} \mathrm{CFU} / \mathrm{ml}\right)$ were found to be lower than that of control $\left(2225 \times 10^{3} \mathrm{CFU} / \mathrm{ml}\right)$. To the best of our knowledge, this is the first report on the antibiofilm activity of M. malabathricum. The result from this study reveals the potential of this medicinal plant to inhibit the formation of $P$. aeruginosa biofilm. 